Original paper

\title{
Potential pitfalls of the PTV concept in dose-to-medium planning optimization
}

\section{E. Sterpin*}

Katholieke Universiteit Leuven, Department of Oncology, OEN I Herestraat 49, 3000 Leuven, Belgium

Université catholique de Louvain, Center of Molecular Imaging, Radiotherapy and Oncology, Institut de recherche expérimentale et clinique, Avenue Hippocrate 54, 1200

Brussels, Belgium

\section{A R T I C L E I N F O}

\section{Article history:}

Received 12 May 2016

Received in Revised form 9 August 2016

Accepted 11 August 2016

Available online $\mathrm{xxxx}$

\section{Keywords:}

Dose to medium

Treatment planning

Dose calculation

Treatment optimization

\begin{abstract}
A B S T R A C T
In typical treatment planning of 3D IMRT, the incident energy fluence is optimized to achieve a homogeneous dose distribution to the PTV. The PTV includes the tumour but also healthy tissues that may have a different dose response for the same incident energy fluence, like bony structures included in the PTV (mandibles in head and neck tumours or femoral bones in sarcomas). Dose to medium optimization compensates for this heterogeneous response, leading to a non-homogeneous energy fluence in the PTV and a non-homogeneous dose in the CTV in the presence of geometric errors. We illustrate qualitatively this statement in a cylindrical geometry where the PTV includes a CTV $(7 \mathrm{~cm}$ diameter) made of water surrounded by ICRU compact bone $(1.2 \mathrm{~cm}$ thickness); such configuration was chosen to exaggerate the aforementioned effect. Optimization was performed assuming dose equals photon energy fluence times mass energy absorption coefficient. Bone has a 4\% lower dose response in a 6 MV flattening filter free spectrum. After optimization either in medium or assuming everything as water composition, the geometry was shifted by $1.2 \mathrm{~cm}$ and dose recomputed. As expected, compensating for the under-response of the bone material during optimization in medium leads to an overdosage of the CTV when patient geometric errors are taken into account. Optimization in dose assuming everything as water composition leads to a uniform coverage. Robust optimization or forcing a uniform atomic composition in the PTV margin may resolve this incompatibility between the PTV concept and dose to medium optimization.
\end{abstract}

(c) 2016 Associazione Italiana di Fisica Medica. Published by Elsevier Ltd. All rights reserved.

\section{Introduction}

The majority of radiotherapy treatments are delivered using external photon beams. In such treatments, the primary aim is typically to deliver a well-defined dose distribution to tumour volumes, even in the presence of systematic and random geometric uncertainties. Dose to organs at risk can be optimally reduced with Intensity Modulated Radiation Therapy (IMRT) while keeping adequate homogeneity to the target volumes.

Most of past and current external radiotherapy has been based on dose engines that transport particles and score dose in water with scaled electronic density according to CT data. These algorithms are usually based on convolution/superposition (C/S) methods that use energy-deposition kernels computed in water or derived from measurements in water [1-5]. The issues with the approximations in the physics of transport inherent to those algorithms have been extensively investigated [1,2,6-9]. In gen-

* Address: Katholieke Universiteit Leuven, Department of Oncology, O\&N I Herestraat 49, 3000 Leuven, Belgium.

E-mail address: edmond.sterpin@kuleuven.be eral, the most significant inaccuracies occur in inhomogeneities where lateral charged particle equilibrium (CPE) is not realized.

However, there is a global increase of the algorithms having the capability to compute dose to medium. Depending on the CT conversion scheme, some convolution/superposition algorithms have already been computing dose to medium through a global scaling of the kernels with the ratio of mass energy absorption coefficients of the considered medium and of water (for instance, the algorithm developed by Ahnesjö [10]. A recent algorithm released by Varian, called Acuros $^{\circledR} \mathrm{XB}$, that solves the linear Boltzmann transport equation, is also able to compute dose to medium at clinically compatible speeds $[11,12]$. This algorithm will likely be used by a significant proportion of radiotherapy centres because of the widespread use of its associated treatment planning system (TPS) $\left(\right.$ Eclipse $^{\circledR}$ ). Monte Carlo simulations (MC) perform more realistic and accurate dose computations [1,2]. The advent of fast MC dose computation engines has enabled the introduction of MC-based algorithms in clinical practice.

The steady increase in availability of dose computation algorithms capable of scoring dose to medium may lead to significant changes in clinical practice. Clinical practice is generally based on dose to water, 
mainly because of historical reasons: (1) reference dosimeters are calibrated in water; (2) clinical trials have been performed using dose calculation algorithms linked to these reference dosimeters and that score dose to water. The advantages and disadvantages of both approaches have been previously discussed in literature [1-5].

We consider the particular but yet typical case of a clinical target volume (CTV) made of water-equivalent tissue. To ensure adequate irradiation of the CTV in the presence of geometric errors, a planning target volume (PTV) is mostly used in current treatment practice. The PTV may include tissues that have different atomic composition than the CTV. For pedagogical reasons and for the sake of simplicity, we consider the case of a homogeneous dose prescription to the PTV. The arguments developed remain valid for heterogeneous prescriptions and will be discussed in the Section 4.

With most conventional algorithms, only the variation of electronic density is taken into account. Hence, all tissues in the PTV are assumed to respond identically to the same energy fluence. To achieve a uniform dose in the whole PTV, the local energy fluence should also be uniform in the PTV. In the presence of geometric uncertainties, the CTV moves in a homogeneous energy fluence field (assuming no modification of the energy fluence distribution because of the geometric uncertainties). Provided that the PTV margins are large enough, the CTV receives a uniform dose. This is not the case if the IMRT treatment is optimized with a dose to medium engine (for instance MC or Acuros). To achieve a uniform dose in the PTV, the optimizer needs to modify the energy fluence in order to compensate the differential response of the different materials. As a consequence, a moving CTV will not be in a homogeneous energy fluence field and thus will not receive a homogeneous dose, which is here the goal of the considered treatment.

The interplay between dose computed in the medium and patient geometric uncertainties (patient motion in the broad sense) has only been sparsely mentioned [1] and never addressed to full extend. In fact, there are intrinsic incompatibilities between the PTV concept and dose to medium optimization leading to the aforementioned dose heterogeneities in the CTV when geometric uncertainties are taken into account. The present study aims at illustrating qualitatively this statement using a simple phantom example with a water-like CTV surrounded by healthy cortical bone. Such phantom configuration was chosen to exaggerate the aforementioned effect. However, the presence of healthy bone in the PTV is relatively common (mandibles for oropharyngeal tumours, vertebras for spinal cord irradiation...).

\section{Material and methods}

\subsection{The PTV concept}

We describe here the PTV concept in the specific context of the margin recipe derived by van Herk et al. [13]. The general formula provided by van Herk et al. to compute a 3D margin $\mathbf{M}_{P T V}$ is of the form:

$\mathbf{M}_{P T V}=\alpha \mathbf{\Sigma}+\beta\left(\boldsymbol{\sigma}-\boldsymbol{\sigma}_{p}\right)$

where $\boldsymbol{\Sigma}, \boldsymbol{\sigma}$ and $\boldsymbol{\sigma}_{p}$ are the vectors of standard deviations for systematic errors, random errors and the penumbra, respectively. The penumbra $\sigma_{p}$ is included as the other random errors in quadrature in $\boldsymbol{\sigma}$. The parameters $\alpha$ and $\beta$ can be adapted depending on the clinical objective. If the objective is to ensure at least $95 \%$ of the prescribed dose to all points of the CTV for $90 \%$ of the patients, then $\alpha=2.5$ and $\beta=1.64$.

The margin recipe relies on several hypotheses, among which:

- the dose distribution is perfectly conformal and spherical,

- the various errors considered obey to Gaussian distributions or have similar standard deviations such that the central limit theorem applies,
- there is shift invariance of the dose distribution.

Strictly speaking, because of the heterogeneous response of the different materials in the PTV, the last hypothesis is violated in reality. The workflow proposed below illustrates that issue.

\subsection{General workflow}

Fig. 1 illustrates comprehensively the workflow of the present study. The geometry is defined in part 1 . The optimization and dose calculation processes are illustrated in parts 2 and 3, respectively. The final result that establishes the main point of this communication is shown in part 4 . All the parts of Fig. 1 are detailed in the following sections.

\subsection{Test geometry}

The test geometry is illustrated in Fig. 1, part 1. A cylindrical CTV of $7 \mathrm{~cm}$ diameter of water material is surrounded by ICRU compact bone material with $1.2 \mathrm{~cm}$ thickness and mass density overridden to $1 \mathrm{~g} / \mathrm{cm}^{3}$ to isolate the effect of atomic composition. The union of the CTV and the $1.2 \mathrm{~cm}$ extension makes the PTV. In the longitudinal direction of the cylinder, we consider that the volumes are infinite, allowing the computation of the dose volumehistograms (DVHs) in an arbitrary transverse plane.

\subsection{Analytical dose optimization and computation}

If the photon energy fluence is perfectly uniform and CPE is established in medium, then dose to medium is given by the formula:

$D_{m}=\Psi\left(\frac{\overline{\mu_{e n}}}{\rho}\right)_{m}$

Let us consider a small water cavity in a medium $\mathrm{m}$ where CPE is established (in medium). According to Siebers et al. [4] and Bragg Gray cavity theory, the dose to water is linked to dose to medium through the formula:

$\frac{D_{w}}{D_{m}}=\frac{\left(\frac{s}{\rho}\right)_{w}}{\left(\frac{s}{\rho}\right)_{m}}=\left(\frac{S}{\rho}\right)_{m}^{w}$

where $\left(\frac{s}{\rho}\right)_{w}$ and $\left(\frac{s}{\rho}\right)_{m}$ are unrestricted mass stopping powers of water and medium, respectively, averaged over the local energy spectra of electrons.

However, as reported by Ma and Li [5], most conventional algorithms compute the dose assuming the patient made of water with variable electronic density using energy kernels computed in water or derived from measurements made in water. Therefore, considering the same hypothesis as for Eq. (1), the dose computed by conventional algorithms is given by:

$D_{\rho_{e l} w}=\Psi\left(\frac{\overline{\mu_{e n}}}{\rho}\right)_{w}$

The notation $D_{\rho_{e l} w}$ is used to distinguish the value computed in Eq. (3) (dose assuming the patient made of water with variable electronic density) from the value computed in Eq. (2) (dose to water converted using stopping power ratios in the sense of Siebers et al. [4]). These two quantities are sometimes mixed in popular perception. The confusion has been cleared recently by $\mathrm{Ma}$ and $\mathrm{Li}$ [5] that states that dose assuming the patient made of water with variable density is closer to dose to medium than dose to water converted using stopping power ratios. This is due to the 
1. Test geometry

2. Optimization in the PTV

$$
\Psi_{W}=\frac{20 G y}{\left(\frac{\bar{\mu}_{e n}}{\rho}\right)_{w}} \quad \Psi_{b}=\frac{20 G y}{\left(\frac{\overline{\mu_{e n}}}{\rho}\right)_{b}}
$$

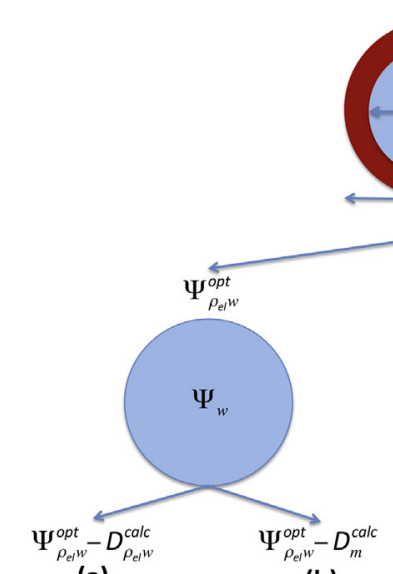

3. Dose computation in the PTV

4. Dose computation in the shifted CTV (a)

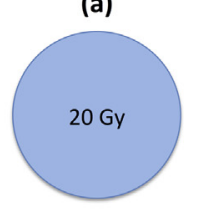

(b)

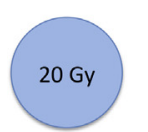

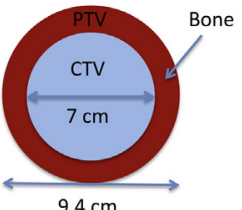

$9.4 \mathrm{~cm}$

\section{e}
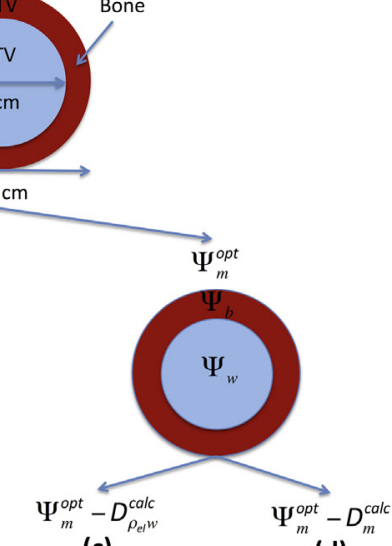

(d)

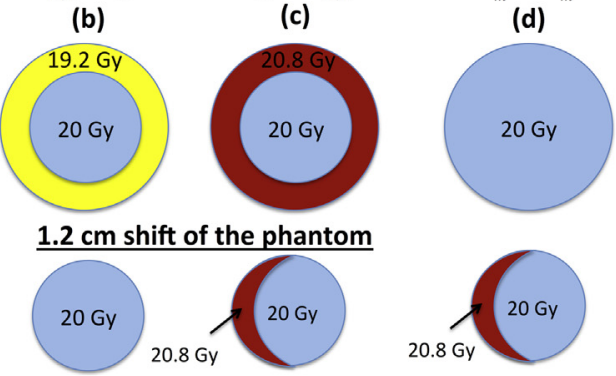

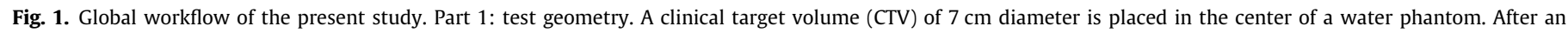

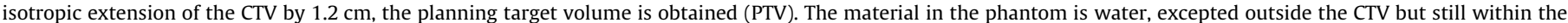

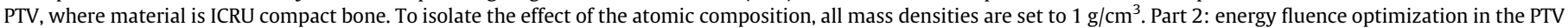

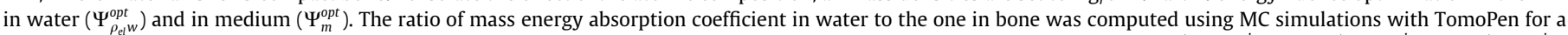

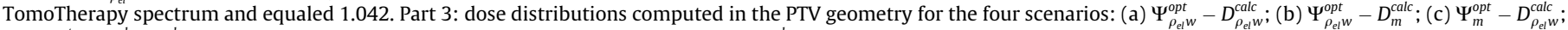

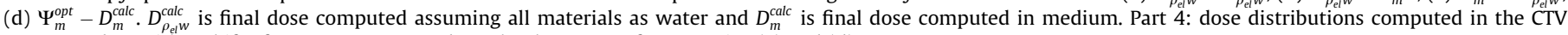
geometry, but with a shift of $1.2 \mathrm{~cm}$. An excess dose clearly appears for scenarios (c) and (d).

fact that mass energy absorption coefficients vary less than stopping powers from one material composition to another in human tissues at clinical energies.

In the PTV, homogeneous dose distributions can be achieved with good approximation using advanced IMRT delivery like TomoTherapy and VMAT. Although a previous paper demonstrated that strict CPE cannot be achieved in radiotherapy [14], we consider that the photon energy fluence is uniform across the PTV in good approximation and that the photon energy fluence is extended sufficiently beyond the PTV margin to compensate for the penumbra. In such conditions, CPE is approximately established and Eqs. (1) and (3) apply. Therefore, the ratio between dose computed by a dose to medium engine and a conventional algorithm is given by:

$\frac{D_{\rho_{e l} w}}{D_{m}}=\frac{\left(\overline{\frac{\mu_{e n}}{\rho}}\right)_{w}}{\left(\frac{\bar{\mu}_{e n}}{\rho}\right)_{m}}=\left(\frac{\overline{\mu_{e n}}}{\rho}\right)_{m}^{w}$

A realistic ratio of Eq. (4) was computed using a previously validated model for TomoTherapy (TomoPen [15-17]). Two central axis depth-dose distributions were computed in a cubic $20 \times 20 \times 20 \mathrm{~cm}^{3}$ phantom and a $5 \times 5 \mathrm{~cm}^{2}$ TomoTherapy static field (resolution $1 \times 1 \times 0.2 \mathrm{~cm}^{3}$ ). The results of the simulations are shown in Fig. 2. The density was set to $1 \mathrm{~g} / \mathrm{cm}^{3}$ but the material was either water or bone. The ratio (2) was computed at $d_{\max }$ to ensure CPE with negligible effect of the attenuation of the beam. The obtained ratio was $104.2 \%$ at $0.3 \%$ statistical uncertainty (one standard deviation). This value of $104.2 \%$ is kept thorough this paper.

The simple analytical expressions (1) and (3) for dose computation allow straightforward optimization of the local fluence depending on the local material. 4 scenarios were envisaged:
1. $\Psi_{\rho_{e l} w}^{\text {opt }}-D_{\rho_{e l} w}^{\text {calc }}$ : optimization was performed to achieve $20 \mathrm{~Gy}$ in the PTV assuming water everywhere with scaled electronic density and final dose calculation was performed assuming water everywhere with scaled electronic density.

2. $\Psi_{\rho_{e l} w}^{\text {opt }}-D_{m}^{\text {calc }}$ : optimization was performed to achieve $20 \mathrm{~Gy}$ in the PTV assuming water everywhere with scaled electronic density and final dose calculation was performed in medium.

3. $\Psi_{m}^{\text {opt }}-D_{\rho_{e l} w}^{\text {calc }}$ : optimization was performed to achieve $20 \mathrm{~Gy}$ (in medium) in the PTV and final dose calculation was performed assuming water everywhere with scaled electronic density.

4. $\Psi_{m}^{\text {opt }}-D_{m}^{\text {calc }}$ : optimization was performed to achieve $20 \mathrm{~Gy}$ (in medium) in the PTV and final dose calculation was performed in medium.

The optimization was drastically simplified. Basically, the optimization process consisted in scaling locally the energy fluence to compensate for the different dose response. For dose to water optimization (scaled electronic density), the fluence was the same everywhere in the PTV. For dose to medium optimization, the fluence was multiplied in the region with compact bone material by the ratio of Eq. (4) (Fig. 1 part 2).

For the CTV and PTV contours at their nominal position, DVHs to the PTV were computed for all four scenarios. To evaluate the effect of a systematic shift of the target volumes, DVHs were computed on a CTV shifted by $1.2 \mathrm{~cm}$ for the 4 scenarios (Fig. 1 part 4 ). Since the clinical objective is to cover the CTV even in the presence of geometric errors, the dose needs to be reported on the CTV when geometric errors are simulated. The reference was the dose computed for the first scenario, which is equivalent to a situation where there are no inhomogeneities. 


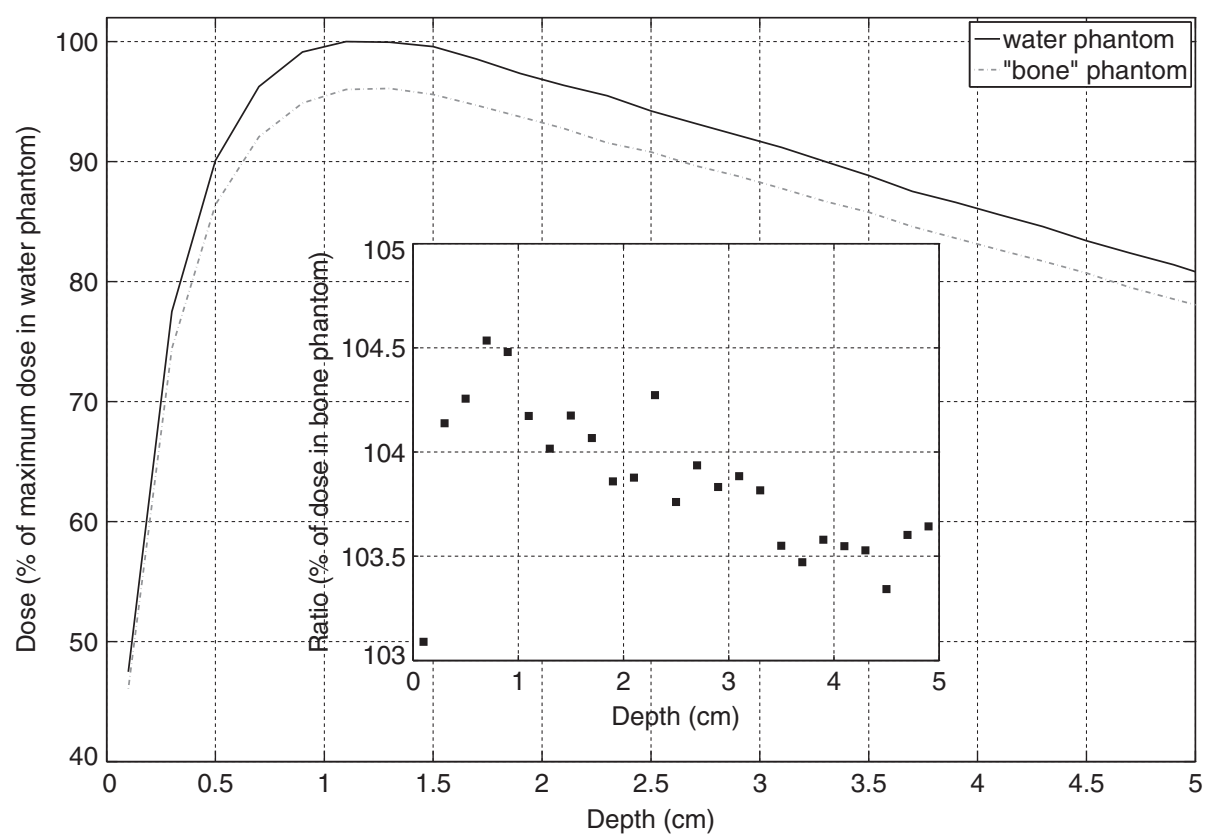

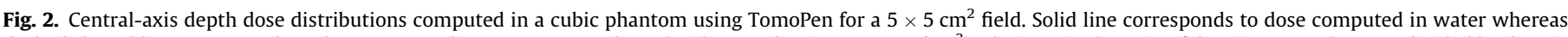

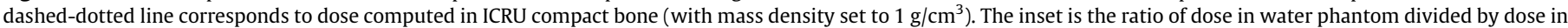
bone phantom (given in \% of dose in bone phantom). Statistical uncertainties were kept below $0.3 \%$ (one standard deviation).

\section{Results}

Fig. 1, part 3 shows dose distributions computed in the PTV geometry of Fig. 1, part 1, according to the four scenarios described in Section 0. Fig. 3(a) shows DVHs in the PTV for the four scenarios. According to Fig. 1 part 3, perfect homogeneity is achieved for $\Psi_{\rho_{e l} w}^{\text {opt }}-D_{\rho_{e l} w}^{\text {calc }}$ and $\Psi_{m}^{\text {opt }}-D_{m}^{\text {calc }}$. There is underdosage for $\Psi_{\rho_{e l} w}^{\text {opt }}-D_{m}^{\text {calc }}$ and overdosage for $\Psi_{m}^{\text {opt }}-D_{\rho_{e l} W^{\text {calc }}}^{\text {calc }}$. These under and over dosages concern roughly $50 \%$ of the PTV volume.

Fig. 1 part 4 shows the dose distributions computed for the CTV geometry as in Fig. 1, but shifted by $1.2 \mathrm{~cm}$, according to the four scenarios described in Section 0. Because the CTV is made of water, the final dose calculation option (water-based or medium-based) does not modify the dose distributions. Only the energy fluence impacts the final result. However, one can notice an overdosage in the CTV for the scenarios with dose to medium optimization $\left(\Psi_{m}^{o p t}\right)$. To compensate for the under-response of the bone material, an extra energy fluence is delivered to the PTV region outside the CTV. However, when the phantom is shifted, this extra fluence impacts a water material, leading to an overdosage in the scenarios $\Psi_{m}^{\text {opt }}-D_{\rho_{e l} w}^{\text {calc }}$ and $\Psi_{m}^{\text {opt }}-D_{m}^{\text {calc }}$. The overdosage concerns roughly $25 \%$ of the CTV, as shown in the DVHs in Fig. 3(b).

\section{Discussion}

With the phantom configuration shown in Fig. 1, the conclusion that can be drawn from Fig. 3 is straightforward. Dose to medium optimization in a heterogeneous PTV leads to a heterogeneous dose to the CTV in the presence of geometric uncertainties. This does not happen with optimization in dose assuming everything as water with scaled electronic density.

Before discussing the clinical implications of these observations, we need first to discuss whether all the hypotheses made may compromise the validity of the conclusions, for instance: (1) the phantom configuration does not necessarily represent a realistic clinical situation; (2) the analytical model is simplistic; (3) density effects have been discarded; (4) only homogeneous dose prescriptions have been considered.
To our knowledge, there is no example of a tumour completely surrounded by compact bone. Due to the significant percentage of calcium, compact bone is the tissue type with the ratio of mass absorption coefficients with respect to water having potentially the largest difference with respect to unity at clinical energies (Eq. (4)). In fact, for $1.5 \mathrm{MeV}$ photons (average of TomoTherapy spectrum [18]), it is the only tissue with a difference to unity larger than $1 \%$ according to data listed in ICRU report 44 . There are though examples where significant amount of healthy bone is included in the PTV: cerebrospinal tumour that is surrounded by the vertebra, sarcomas close to the femoral bone, head and neck tumours with inclusion of the mandible in the PTV etc. Despite these examples, the phantom design chosen for this study is not representative of the majority of the treatments and should be considered for illustration purposes only. However, the whole argument remains the same if only one single voxel in the PTV margin had a material with a response significantly different than the tissues included in the CTV. A dose to medium optimizer would try to compensate the differential response in that voxel. Such compensation will have no clinical impact because it concerns a single voxel, but this does not justify attempting to compensate the differential response in a first place.

The analytical model is indeed simplistic because it neglects the potential influence of the electrons. To evaluate the impact of the approximations of the model, a MC study has been conducted in parallel that reproduces the workflow presented in Fig. 1. The methodology and the results of the MC study are presented in the appendix. Similar effects were observed and led to the same conclusions.

Density effects have been deliberately neglected by setting all mass densities to unity. This was necessary to isolate the effect of atomic composition on dose distributions. However, the PTV concept shows also limitations to handle healthy tissues included in the PTV that have a different density than the tumour (for instance, lung tissues). IMRT optimization with advanced algorithms that take into account loss of electronic equilibrium may lead to similar inconsistencies than the ones presented in this study for dose to medium optimization, as illustrated by Lacornerie et al. [19] for lung tumours. Air cavities included in the PTV may also interfere negatively with the optimization process as they combine both a 


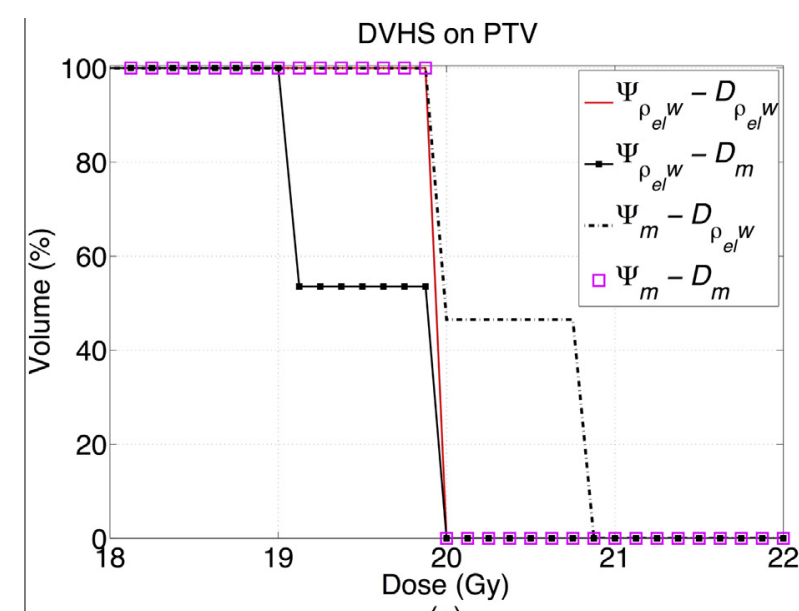

(a)

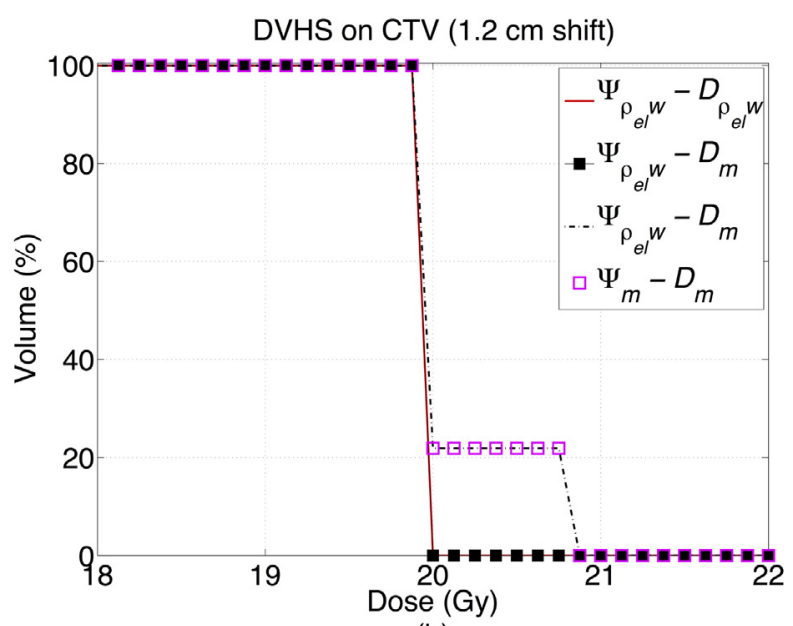

(b)

Fig. 3. Dose volume histograms in the PTV (a) at nominal position and the CTV (b) with $1.2 \mathrm{~cm}$ shift as shown in Fig. 1 for the four scenarios: (red continuous line) $\Psi_{\rho_{e l} w}^{o p t}-D_{\rho_{e l} w}^{\text {calc }}$; (black line + squares) $\Psi_{\rho_{e l} w}^{\text {opt }}-D_{m}^{\text {calc }}$; (gray dashed-dotted) $\Psi_{m}^{\text {opt }}-D_{\rho_{e \mid l}{ }^{\text {calc }}}$; (magenta open squares) $\Psi_{m}^{\text {opt }}-D_{m}^{\text {calc }}$. $\Psi_{\rho_{e l} w}^{\text {opt }}$ is optimization in dose assuming everything as water with scaled electronic density; $D_{\rho_{e l} w}^{\text {calc }}$ is final dose computed assuming everything as water with scaled electronic density; $\Psi_{m}^{o p t}$ is optimization in dose to medium; $D_{m}^{\text {calc }}$ is final dose computed in medium. (For interpretation of the references to color in this figure legend, the reader is referred to the web version of this article.)

low density and a significantly different atomic composition than water (difference of order of $10 \%$ for mass energy absorption at $1.5 \mathrm{MeV}$ photon energy and $12 \%$ for mass stopping power with respect to water for a $6 \mathrm{MV}$ beam). Attempting to compensate during IMRT optimization for the different dose response in air to achieve a homogeneous dose to the PTV may lead to considerable distortions of the energy fluence and thus undesired dose distributions to the CTV when geometric errors are taken into account.

Finally, only homogeneous prescriptions have been considered. Homogeneous prescription is standard for most treatment strategies but some of them like stereotactic body radiation therapy (SBRT) rely on heterogeneous prescriptions instead. However, the conclusions of the present study hold for heterogeneous prescriptions as well. Dose to medium optimization will compensate the differential dose responses of the healthy tissues included in the PTV to match the dose prescription, leading to distortions of the energy fluence and an unjustified discrepancy between the expected dose and the delivered dose to a moving CTV.

The incompatibility between the PTV concept and dose to medium optimization is magnified if dose to medium is converted into dose to water using stopping power ratios (Eq. (1)). For the same energy fluence, the difference between dose to water "converted" and dose assuming everything as water with scaled electronic density is around $11 \%$ for compact bone [4] . If an optimizer based on "dose to water converted with stopping power ratios" is used, the heterogeneities in the fluence distribution will be larger than using dose to medium optimization only.

Only the case of photons was considered. However, the argument applies also to electrons and heavy charged particles like protons. The main difference is that the ratio between dose to water with scaled electronic density (or better, dose assuming everything as water with correct water equivalent pathlength) and dose to medium is better approximated by Eq. (1) than Eq. (4) (for electrons, this is true for shallow depths only, as explained in [5]). Thus, dose in the PTV should be ideally optimized assuming everything in the PTV as water with correct water equivalent pathlength to avoid undesired heterogeneities in the dose distribution in the CTV when geometric uncertainties are taken into account.

Should we conclude dose to medium optimization should be discarded? In fact, dose to medium optimization introduces a refinement which current treatment practice is not completely adapted for. In the vast majority of cases, the actual objective of treatment planning is that the CTV moves within an homogeneous energy fluence that leads for all points in the CTV to an homogeneous dose as prescribed by the physician (assuming a CTV responding uniformly). The PTV concept has been invented to satisfy this objective. The PTV assumes inherently a uniform response of all points in the PTV, which is an approximation. However, this approximation is consistent with the approximation of assuming everything as water with scaled electronic density for dose calculation, leading to homogeneous dose distributions in the CTV even in the presence of geometric errors (scenarios $\Psi_{\rho_{e l} w^{\text {opt }}}-D_{\rho_{e l}{ }^{\mathrm{w}}}^{\text {calc }}$ and $\Psi_{\rho_{e l}{ }^{w}}^{\text {opt }}-D_{m}^{\text {calc }}$ ). Dose to medium optimization is more accurate on a physical point of view, but it breaks down this consistency as observed for scenarios $\Psi_{m}^{\text {opt }}-D_{\rho_{e l} w}^{\text {calc }}$ and $\Psi_{m}^{\text {opt }}-D_{m}^{\text {calc }}$.

An elegant way to solve this inconsistency is to use a comprehensive robust optimizer [20,21], which avoids the need of a PTV. Every iteration (or once every $\times$ iteration) of the optimizer, dose to medium distribution should be computed taking into account explicitly random and systematic errors. For instance, breathing motion can be simulated by computing dose distributions on a 4D CT scan. Systematic setup errors can be taken into account by computing dose distributions for several scenarios (patient systematically shifted to the left, or to the right etc.). Since only the dose to the CTV is considered, it is possible to deliver a uniform dose to the CTV in the presence of geometric errors even for an extreme phantom design as in Fig. 1.

Such methodology is extremely demanding computationally and will likely not be widely available in the short future. Optimization with dose to water with scaled electronic density should still be considered as a good alternative as it allows for an accurate modelling of attenuation while avoiding any unjustified distortion of the energy fluence. If dose to medium optimization is ultimately used, a pragmatic solution would be to force the atomic composition within the PTV margin to be the same as the tumour tissue (while keeping the correct electronic density). Such strategy will avoid unnecessary heterogeneities in the local fluence, no matter the particle considered.

\section{Conclusions}

For the specific context of a PTV that includes healthy tissues in the PTV margin that respond differently than the tissues making the CTV, dose to medium optimization should be introduced cau- 

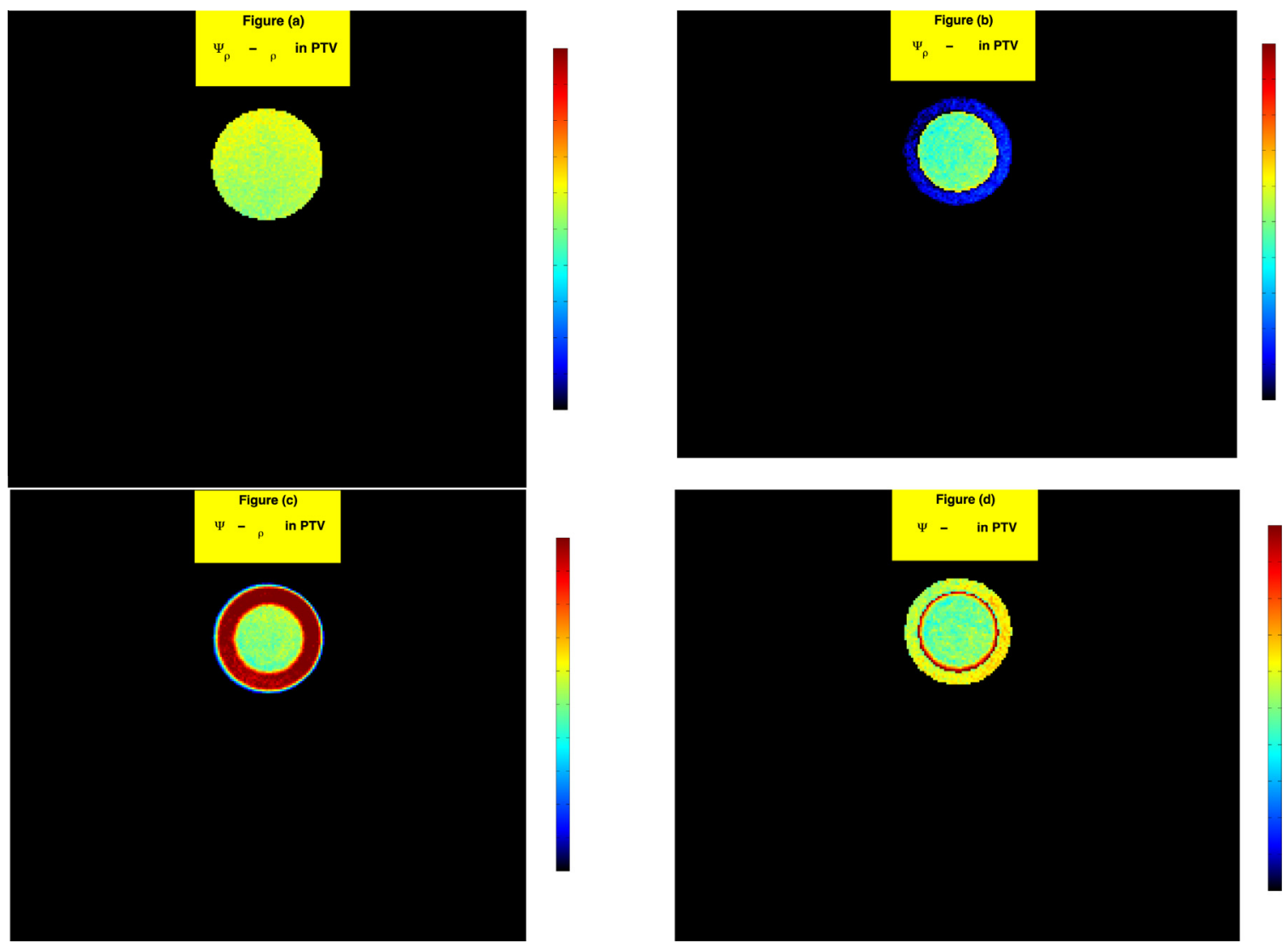

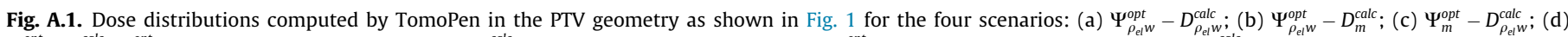

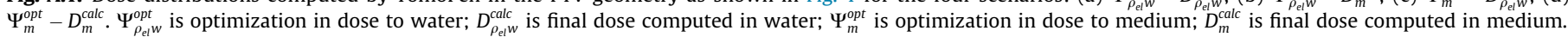

tiously. Dose to medium optimization compensates for the heterogeneous response of the various tissues in the PTV, leading to nonhomogeneous energy fluences in the PTV and thus non-

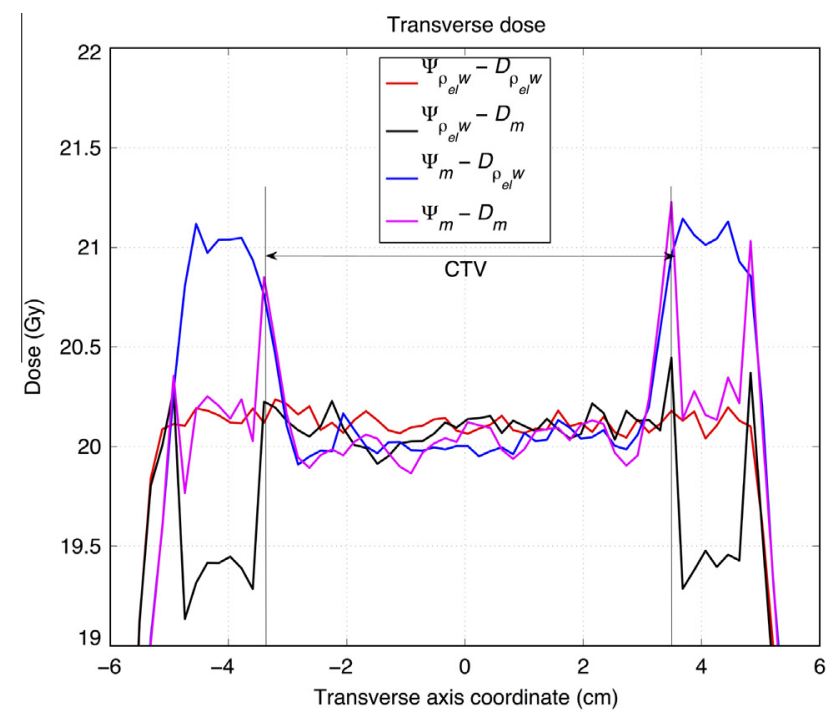

Fig. A.2. Dose profiles computed by TomoPen and acquired through the center of the PTV as shown in Fig. 1 for the four scenarios: (red) $\Psi_{\rho_{\text {l }} w}^{\text {opt }}-D_{\rho_{\mathrm{e} \text { w }} \text {; }}^{\text {calc }}$ (black line) $\Psi_{\rho_{e l} w}^{o p t}-D_{m}^{\text {calc }}$; (blue) $\Psi_{m}^{\text {opt }}-D_{\rho_{e l} w}^{\text {calc }}$; (magenta) $\Psi_{m}^{\text {opt }}-D_{m}^{\text {calc }} . \Psi_{\rho_{e l} w}^{\rho_{e l} w}$ is optimization in dose to water; $D_{\rho_{e l} \text { cal }}$ is final dose computed in water; $\Psi_{m}^{\text {opt }}$ is optimization in dose to

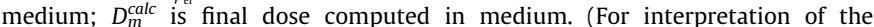
references to color in this figure legend, the reader is referred to the web version of this article.) homogeneous dose distributions in the CTV in the presence of geometric uncertainties.

The most comprehensive way to cope with this phenomenon is to implement comprehensive robust optimization. Such solution is not straightforward and computationally demanding. A more immediate solution would be to force all tissue compositions in the PTV margin to match tumour tissue composition (while keeping the correct electronic density).

\section{Acknowledgements}

Hugo Palmans, Nick Reynaert and Hugo Bouchard are particularly acknowledged for their useful comments on the manuscript. The author would like to thank Stefaan Vynckier, John Lee, Guillaume Janssens, Jonathan Orban, Jefferson Sorriaux and Kevin Souris for their stimulating discussions.

\section{Appendix. Assessment of the effect of dose to medium optimization using MC simulations and the TomoTherapy system}

In this appendix, we repeat the study using the geometry in Fig. 1 with the TomoTherapy system and the MC model TomoPen validated elsewhere [19-21] instead of our simple analytical approach. The PTV geometry was contoured in the standard cylindrical Virtual Water ${ }^{\mathrm{TM}}$ phantom provided with every TomoTherapy unit. The extension of the CTV in the longitudinal direction equaled $10 \mathrm{~cm}$. In order to keep similar the total attenuation coefficient between the water and the bone materials, the mass density of 


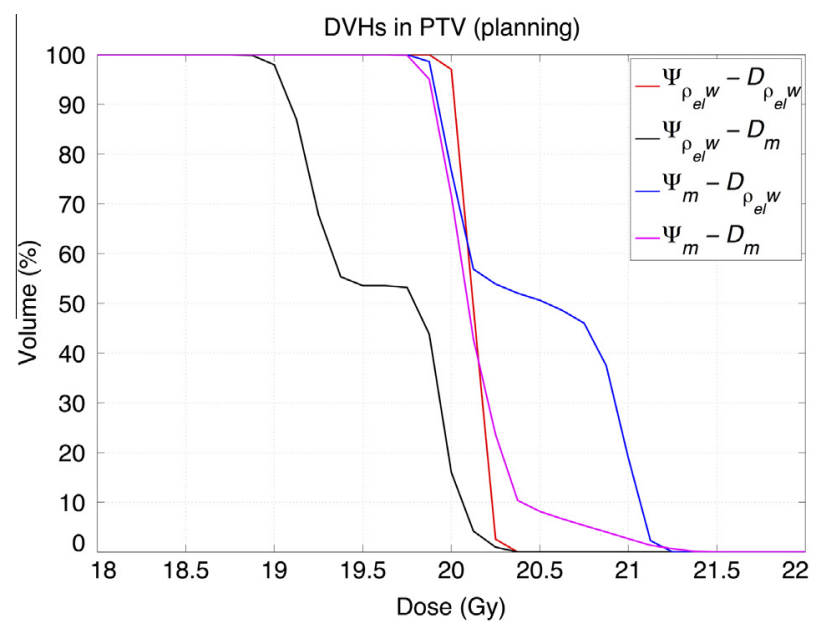

Fig. A.3. Dose volume histograms of the dose distributions computed by TomoPen in the PTV shown in Fig. 1 for the four scenarios: (red) $\Psi_{\rho_{e} w}^{\text {opt }}-D_{\rho_{e l} w}^{\text {calc }}$; (black line) $\Psi_{\rho_{e l} W}^{\text {opt }}-D_{m}^{\text {calc }}$; (blue) $\Psi_{m}^{\text {opt }}-D_{\rho_{e l} w}^{\text {calc }}$; (magenta) $\Psi_{m}^{\text {opt }}-D_{m}^{\text {calc }} . \Psi_{\rho_{e l} w}^{\rho_{e l} w}$ is optimization in dose to water; $D_{\rho_{w}}^{\text {calc }}$ is final dose computed in water; $\Psi_{m}^{\text {opt }}$ is optimization in dose to medium; $D_{m}^{\text {calc }}$ is final dose computed in medium. (For interpretation of the references to color in this figure legend, the reader is referred to the web version of this article.)

ICRU compact bone was set to $1 \mathrm{~g} / \mathrm{cm}^{3}$. For the scenarios $\Psi_{\rho_{e l} w}^{\text {opt }}-D_{\rho_{e l} w}^{\text {calc }}$ and $\Psi_{\rho_{e l}{ }^{\mathrm{ppt}}}^{\text {of }}-D_{m}^{\text {calc }}$, a homogeneous dose was planned to the PTV (20 Gy) using the TomoTherapy system (helical delivery, pitch $=0.43$, planned modulation factor $=3.0$ and slice width $=2.5 \mathrm{~cm}$ ). The final dose calculation was performed by
TomoPen either in water or in medium. The statistical standard deviation was about $1 \%$ in the high dose region. As it can be observed in Figs. A.1(b), A.2, and A.3, there is an underdosage in the bone region for $\Psi_{\rho_{e l} w}^{\text {opt }}-D_{m}^{\text {calc }}$. This underdosage is about $5 \%$, close to the $4 \%$ value computed for the static field (Fig. 2 of the main document).

For the scenarios $\Psi_{m}^{o p t}-D_{\rho_{e l} w}^{\text {calc }}$ and $\Psi_{m}^{\text {opt }}-D_{m}^{\text {calc }}$ the effect of the bone heterogeneity was compensated in the TomoTherapy TPS by prescribing $21 \mathrm{~Gy}$ within the PTV outside of the CTV (same planning parameters). For $\Psi_{m}^{\text {opt }}-D_{m}^{\text {calc }}$ good homogeneity was achieved, as shown in Figs. A.1(d), A.2, and A.3, although there is some overdosage at the limit of the CTV due to the penumbra of the dose distribution provoked by the extra dose delivered to the PTV. However, PTV coverage is much improved in $\Psi_{m}^{\text {opt }}-D_{m}^{\text {calc }}$ compared to $\Psi_{\rho_{e l}{ }^{w}}^{\text {opt }}-D_{m}^{\text {calc }}$

As in the main document, the phantom was shifted by $1.2 \mathrm{~cm}$ and dose distributions were computed again for the four scenarios. Since target coverage of the CTV is the clinical objective in the presence of geometric errors, DVHs were computed in the CTV only. The same conclusions can be drawn from Figs. A.4 and A.5 as from Fig. 3. Almost identical coverage was achieved for the CTV in the scenarios $\Psi_{m}^{\text {opt }}-D_{\rho_{e l} \text { calc }}^{\text {calc }}$ and $\Psi_{\rho_{e l} w}^{\text {opt }}-D_{m}^{\text {calc }}$. However, there is an unnecessary excess dose to the CTV for the scenarios $\Psi_{m}^{\text {opt }}-D_{\rho_{e l} w}^{\text {calc }}$ and $\Psi_{m}^{\text {opt }}-D_{m}^{\text {calc }}$. The reference is $\Psi_{\rho_{e l} w}^{\text {opt }}-D_{\rho_{e l} w}^{\text {calc }}$ (i.e. the ideal case of an homogeneous medium). $D_{50}$ equals $19.9 \mathrm{~Gy}$ for both $\Psi_{\rho_{e l} w}^{\text {opt }}-D_{\rho_{e l} w}^{\text {calc }}$ and $\Psi_{\rho_{e l} w}^{\text {opt }}-D_{m}^{\text {calc }}$. However, for and $\Psi_{m}^{\text {opt }}-D_{m}^{\text {calc }}, D_{50}$ equals $20.2 \mathrm{~Gy}$ (1.5\% overdosage). $D_{2}$ equals 20.5 and $20.3 \mathrm{~Gy}$ for $\Psi_{\rho_{e l} w}^{\text {opt }}-D_{\rho_{e l} w}^{\text {calc }}$
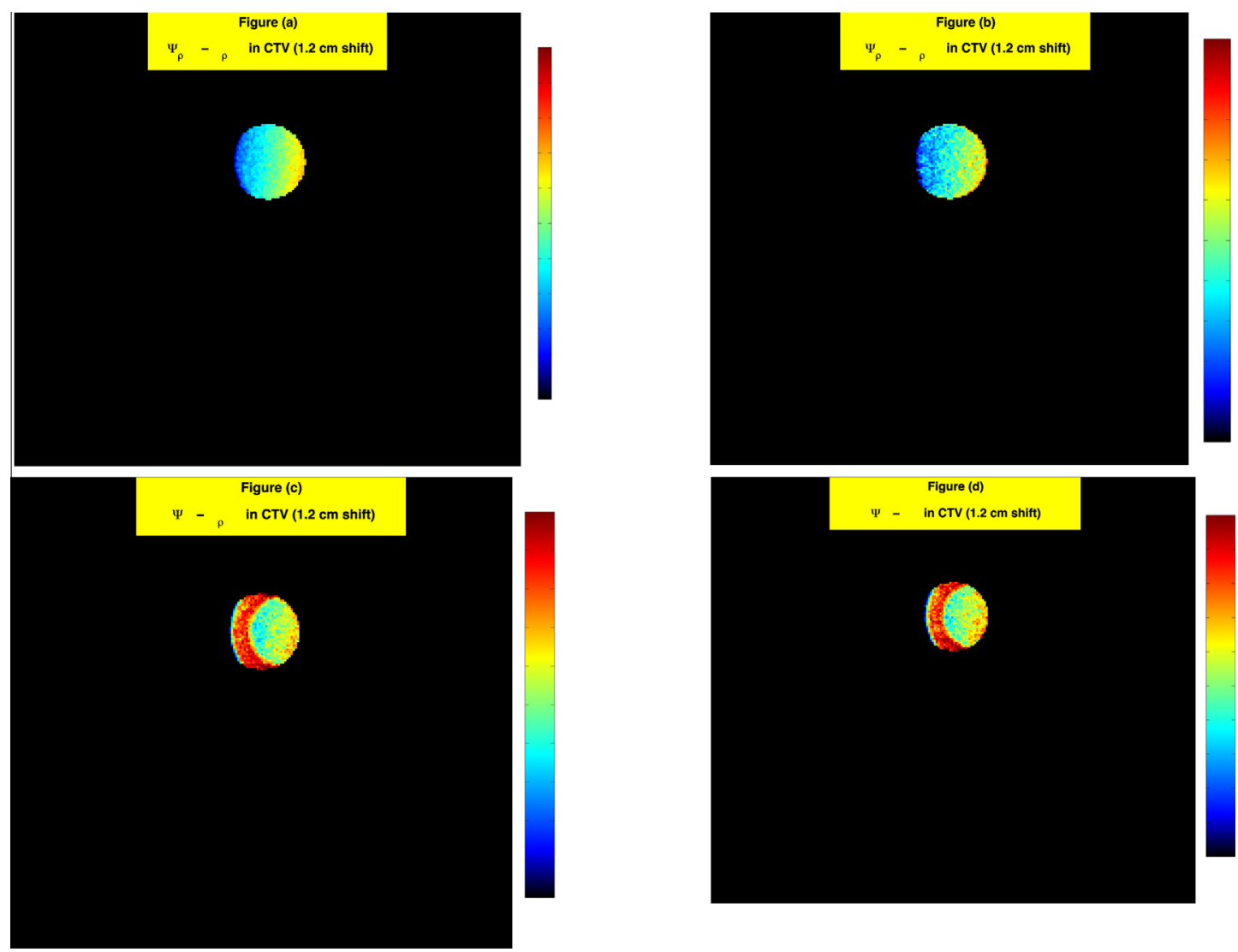

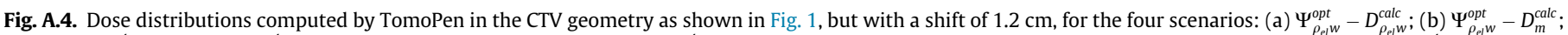

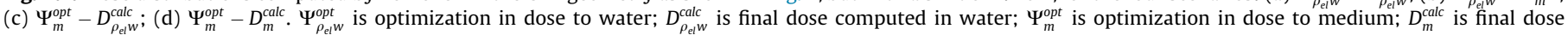
computed in medium. 


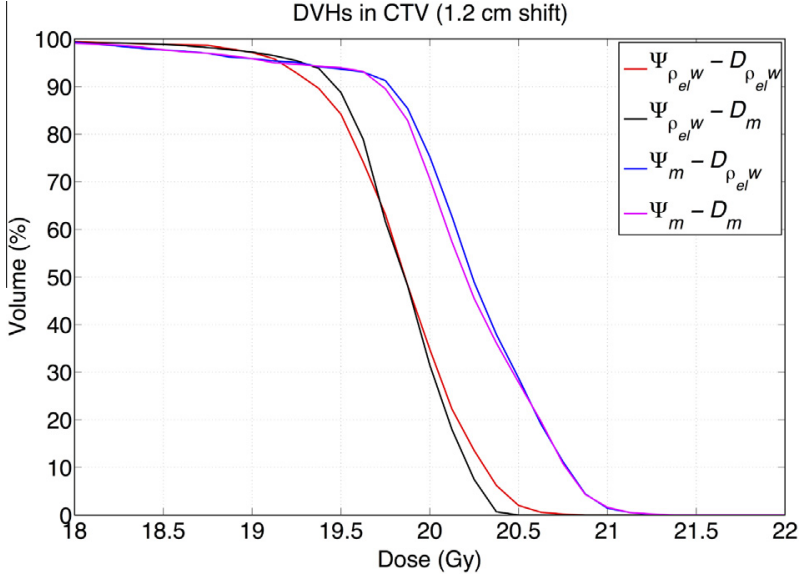

Fig. A.5. Dose volume histograms of the dose distributions computed by TomoPen in the CTV $\left(1.2 \mathrm{~cm}\right.$ shift) shown in Fig. 1 for the four scenarios: (red) $\Psi_{\rho_{e l} \text { opt }}^{\text {ow }}-D_{\rho_{e l} w}^{\text {calc }}$; (black line) $\Psi_{\rho_{e l} w}^{\text {opt }}-D_{m}^{\text {calc }}$; (blue) $\Psi_{m}^{\text {opt }}-D_{\rho_{e l} w}^{\text {calc }}$; (magenta) $\Psi_{m}^{\text {opt }}-D_{m}^{\text {calc }}{ }^{\rho_{e l} w} \Psi_{\rho_{\text {et }} w}^{\text {opt }} \rho_{\text {el }}$ is optimization in dose to water; $D_{\rho_{e l} \text { calc }}$ is final dose computed in water; $\Psi_{m}^{\rho_{e l} w}$ is optimization in dose to medium; $D_{m}^{\text {calc }}$ is final dose computed in medium. (For interpretation of the references to color in this figure legend, the reader is referred to the web version of this article.)

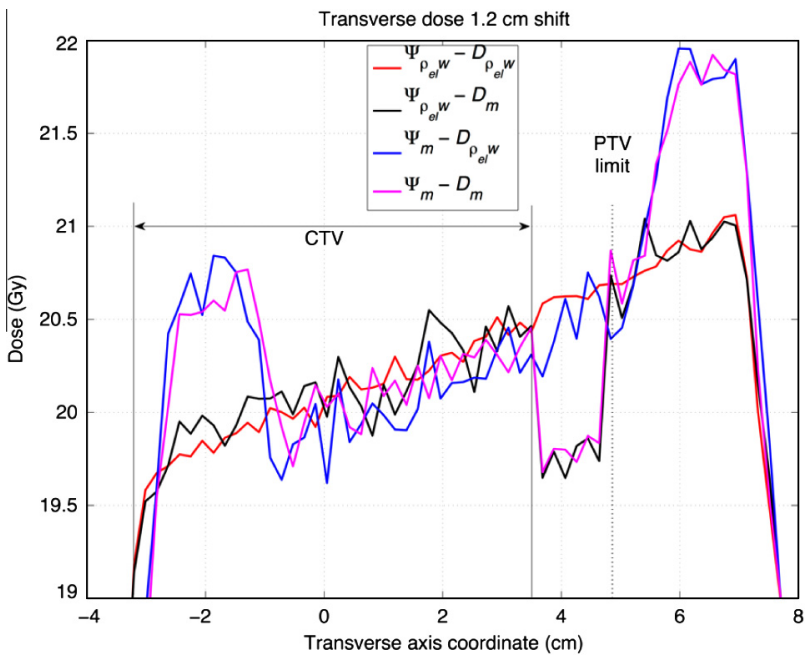

Fig. A.6. Dose profiles computed by TomoPen and acquired through the center of the PTV for the phantom of Fig. 1 shifted by $1.2 \mathrm{~cm}$ for the four scenarios: (red)

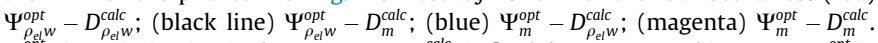
$\Psi_{\rho_{\text {et }} w}^{\rho_{e} w}$ is optimization in dose to water; $D_{\rho_{\rho} w}^{\text {calc }}$ is final dose computed in water; $\Psi_{m}^{\text {opt }}$ is optimization in dose to medium; $D_{m}^{\text {calc }} \rho_{\text {el }}$ is final dose computed in medium. The profiles are acquired in the same direction than the direction of the phantom shift. (For interpretation of the references to color in this figure legend, the reader is referred to the web version of this article.)

and $\Psi_{\rho_{e l} w^{o p t}}^{\text {of }}-D_{m}^{\text {calc }}$, respectively. For both $\Psi_{m}^{\text {opt }}-D_{\rho_{e l} w}^{\text {calc }}$ and $\Psi_{m}^{\text {opt }}-D_{m}^{\text {calc }}$, $D_{2}$ equals $20.9 \mathrm{~Gy}$, thus an overdosage of $1.9 \%$ from the reference $\Psi_{\rho_{e l} w}^{\text {opt }}-D_{\rho_{e l} w}^{\text {calc }}$.

Although the overdosage is rather small, these observations lead to the same conclusions than in the main document where a simple analytical model was used: using $\Psi_{m}^{o p t}$ leads to inhomogeneous dose to the CTV in the presence of geometric errors. Moreover, unnecessary excess dose is also delivered outside the target volumes. Fig. A.6 shows transverse dose profiles acquired through the center of the PTV. One can observe a slight tilt of the dose distributions compared to Fig. A.2 that is due to the modification of the amount of material seen by the photons when the phantom is shifted by $1.2 \mathrm{~cm}$ (violation of the hypothesis of shift invariance of the dose distribution). However, what is important to note is the overdosage of the region right next to the PTV in the scenarios $\Psi_{m}^{o p t}-D_{\rho_{e l} w}^{\text {calc }}$ and $\Psi_{m}^{\text {opt }}-D_{m}^{\text {calc }}$. The overdosage reaches locally $5 \%$, which is consistent with the additional dose delivered to compensate the under-response of the bone material. On a clinical point of view, this overdosage is superfluous.

\section{References}

[1] Chetty IJ, Curran B, Cygler JE, DeMarco JJ, Ezzell G, Faddegon Ba, et al. Report of the AAPM Task Group No. 105: issues associated with clinical implementation of Monte Carlo-based photon and electron external beam treatment planning. Med Phys 2007;34:4818. http://dx.doi.org/10.1118/1.2795842.

[2] Reynaert N, Vandermarck S, Schaart D, Vanderzee W, Vanvlietvroegindeweij C Tomsej $\mathrm{M}$, et al. Monte Carlo treatment planning for photon and electron beams. Radiat Phys Chem 2007;76:643-86. http://dx.doi.org/10.1016/i. radphyschem.2006.05.015.

[3] Liu HH, Keall P, Hendee WR. D[sub m] rather than D[sub w] should be used in Monte Carlo treatment planning. Med Phys 2002;29:922. http://dx.doi.org/ $10.1118 / 1.1473137$.

[4] Siebers JV, Keall PJ, Nahum aE, Mohan R. Converting absorbed dose to medium to absorbed dose to water for Monte Carlo based photon beam dose calculations. Phys Med Biol 2000;45:983-95.

[5] Ma C-M, Li J. Dose specification for radiation therapy: dose to water or dose to medium? Phys Med Biol 2011;56:3073-89. http://dx.doi.org/10.1088/00319155/56/10/012.

[6] Ahnesjö a, Aspradakis MM. Dose calculations for external photon beams in radiotherapy. Phys Med Biol 1999;44:R99-R155.

[7] Carrasco P, Jornet N, Duch Ma, Weber L, Ginjaume M, Eudaldo T, et al Comparison of dose calculation algorithms in phantoms with lung equivalent heterogeneities under conditions of lateral electronic disequilibrium. Med Phys 2004;31:2899. http://dx.doi.org/10.1118/1.1788932.

[8] Arnfield MR, Siantar CH, Siebers J, Garmon P, Cox L, Mohan R. The impact of electron transport on the accuracy of computed dose. Med Phys 2000:27:1266-74.

[9] Sterpin E, Salvat F, Olivera G, Vynckier S. Monte Carlo evaluation of the convolution/superposition algorithm of $\mathrm{Hi}^{-\mathrm{Art}^{\mathrm{TM}}}$ tomotherapy in heterogeneous phantoms and clinical cases. Med Phys 2009;36:1566. http:// dx.doi.org/10.1118/1.3112364.

[10] Ahnesjö A. Collapsed cone convolution of radiant energy for photon dose calculation in heterogeneous media. Med Phys 1989;16:577.

[11] Fogliata A, Nicolini G, Clivio A, Vanetti E, Mancosu P, Cozzi L. Dosimetric validation of the Acuros XB Advanced Dose Calculation algorithm: fundamental characterization in water. Phys Med Biol 2011;56:2885-6. http://dx.doi.org/10.1088/0031-9155/56/9/2885.

[12] Vassiliev ON, Wareing TA, McGhee J, Failla G, Salehpour MR, Mourtada F. Validation of a new grid-based Boltzmann equation solver for dose calculation in radiotherapy with photon beams. Phys Med Biol 2010;55:581-98. http://dx. doi.org/10.1088/0031-9155/55/3/002.

[13] van Herk M, Remeijer P, Rasch C, Lebesque JV. The probability of correct target dosage: dose-population histograms for deriving treatment margins in radiotherapy. Int J Radiat Oncol Biol Phys 2000;47:1121-35.

[14] Bouchard H, Seuntjens J, Palmans H. On charged particle equilibrium violation in external photon fields. Med Phys 2012;39:1473-80. http://dx.doi.org/ $10.1118 / 1.3684952$

[15] Sterpin E, Tomsej M, Cravens B, Salvat F, Ruchala K, Olivera GH, et al. Monte Carlo simulation of the Tomotherapy treatment unit in the static mode using MC HAMMER, a Monte Carlo tool dedicated to Tomotherapy. J Phys Conf Ser 2007;74:1-10. http://dx.doi.org/10.1088/1742-6596/74/1/012019.

[16] Sterpin E, Salvat F, Olivera GH, Vynckier S. Analytical model of the binary multileaf collimator of tomotherapy for Monte Carlo simulations. J Phys Conf Ser 2008;102:012022. http://dx.doi.org/10.1088/1742-6596/102/1/012022.

[17] Sterpin E, Chen Y, Chen Q, Lu W, Mackie TR, Vynckier S. Monte Carlo-based simulation of dynamic jaws tomotherapy. Med Phys 2011;38:5230-8. http:/ dx.doi.org/10.1118/1.3626486.

[18] Sterpin E, Salvat F, Cravens R, Ruchala K, Olivera GH, Vynckier S. Monte Carlo simulation of helical tomotherapy with PENELOPE. Phys Med Biol 2008;53:2161-80. http://dx.doi.org/10.1088/0031-9155/53/8/011.

[19] Lacornerie T, Lisbona A, Mirabel X, Lartigau E, Reynaert N. GTV-based prescription in SBRT for lung lesions using advanced dose calculation algorithms. Radiat Oncol 2014;9:223. http://dx.doi.org/10.1186/s13014-0140223-5.

[20] Gordon JJ, Sayah N, Weiss E, Siebers JV. Coverage optimized planning: Probabilistic treatment planning based on dose coverage histogram criteria. Med Phys 2010;37:550. http://dx.doi.org/10.1118/1.3273063.

[21] Korreman SS. Motion in radiotherapy: photon therapy. Phys Med Biol 2012;57:R161-91. http://dx.doi.org/10.1088/0031-9155/57/23/R161. 